\title{
Ecological strain testing of breeding lines of soft spring wheat in Bagan created on the basis of distant hybridization
}

\author{
Nemtsev B.F. ${ }^{1 *}$, Nemtsev A.B. ${ }^{1}$, Goncharov N.P. ${ }^{2}$, Kurkova S.V. ${ }^{3}$ \\ ${ }^{1}$ Siberian Research Institute of Plant Production and Breeding - Branch of the Institute of Cytology \\ and Genetics, SB RAS, Krasnoobsk, Novosibirsk region, Russia \\ ${ }^{2}$ Institute of Cytology and Genetics, SB RAS, Novosibirsk, Russia \\ ${ }^{3}$ Siberian Federal Scientific Centre of Agro-BioTechnologies, RAS, Siberian Research Institute \\ of Feedstuff, North-Kulunda Department, Novosibirsk region, Russia \\ *e-mail:nembor@yandex.ru
}

The article presents long-term results of studying under the conditions of risky agriculture Kulunda steppe the ecological homeostasis of spring soft wheat breeding lines, created using remote hybridization. The studies were carried out at the breeding and seed sector of grain and feed crops of the SFNTSA RAS Siberian Research Institute of Feed NorthKulunda Department. One of the important indicators characterizing the resistance of plants to adverse environmental factors is homeostasis - a universal property in the system of the relationship between the genotype and the environment. Homeostasis is the ability of the genotype to minimize the effects of adverse external conditions. Statistical processing was performed using a package of Snedecor applications. There were carried on an analysis of the homeostasis of the selection lines according to the algorithms developed by Martynov S.P., an yield variance analysis of multiyear experience according to Tomilov, and multidimensional ranking of varieties on the main breeding traits, which was designed by I.A. Uzhakov. A breeding line (1459-E-06) of spring soft wheat with high responsiveness to the environmental conditions and stable high yield was selected. This line was made by crossing a spelt with a durum wheat and further backcrossing with soft wheat. It gave a significant increase in yield, in relation to the Omsk standard $360.3 \mathrm{t} / \mathrm{ha}$, due to the plants survival. This line is advisable to use as a source of high productivity of plants in the selection process, to obtain new genotypes with high adaptive properties and is recommended for transfer to the state strain testing. 